\title{
Charge transfer control by substituents: Donor pyrroles and fluoro-anilines
}

\author{
Antje Neubauer, Sukumaran Murali, and Wolfgang Rettig \\ Institut für Chemie, Humboldt-Universität zu Berlin, Brook-Taylor-Str. 2, D-12489 Berlin, Germany
}

\begin{abstract}
Derivatives of N-phenyl pyrrole with a para-donor substituent on the acceptor benzene ring are compared. It is shown that by a suitable increase of the donor strength of the pyrrolo group, CT fluorescence can be achieved even for donor-donor substituted benzenes. On the other hand, introducing fluoro substituents into the acceptor moiety strongly enhances the CT formation tendency, and several new compounds with CT fluorescence even in alkane solvents are presented.
\end{abstract}

\section{INTRODUCTION}

The photophysics of donor-acceptor substituted benzenes are of great interest in the study of intramolecular charge transfer (ICT) states. These ICT states can be observed as strongly redshifted and highly solvatochromic fluorescence bands, often leading to dual fluorescence, e.g. in dimethylamino benzonitrile (DMABN) and its derivatives, as well as in N-phenylpyrrole (PB) and its derivatives [1]. Some ICT compounds are connected with allowed emission (high transition dipole moment, $M_{f}$ ), others with forbidden emission (small $M_{f}$ ). The difference between the two types of CT states is the mesomeric interaction between the molecular subsystems: ICT states with allowed emission are generally found in nearly coplanar compounds with high mesomeric interaction (mesomeric ICT state, MICT) [2]. ICT states with forbidden emission and small mesomeric interaction are favoured for perpendicular geometries and are commonly called TICT (Twisted Intramolecular Charge Transfer) states [1, 3, 4].

Recently, theoretical studies on N-pyrrolobenzene (PB), N-pyrrolobenzonitrile (PBN) and 4-N,N-dimethylaminobenzonitrile (DMABN) were performed by Parusel for the ground and excited states using a DFT/MRCI approach. It was found that unlike for DMABN, the lowest TICT state of PB and PBN is of B symmetry and is more stable than the LE state even in non polar solvents for PBN [5].

Later, Zilberg et al. [6] studied the same molecules using excited state optimization in the ab initio CASSCF frame work. Their calculations support two distinct structures (energetic minima) for the CT state. One possesses a quinoid bond length alternation in benzene, with a preferred coplanar arrangement of the chromophores, corresponding to the MICT [2] model as well as the PICT model (planar intramolecular charge transfer) introduced earlier [7, 8]. The other excited state minimum has an antiquinoid bond length distribution in the benzene ring (long central bonds) and a twisted structure connected with a larger dipole moment consistent with the TICT model [1]. In contrast to the Parusel study, these lowest two CT minima were found to be of A symmetry.

\section{EXPERIMENTAL}

The synthesis of the compounds (for structures and abbreviations see Scheme 1) is described in recently published and in forthcoming publications [9-12], as well as more spectral details. Solvents were of Merck Uvasol quality.

Absorption spectra were measured on an ATI UNICAM Series UV-Visible Spectrometer UV4. An AMINCOBowmann series 2 Luminescence spectrometer was used for the measurement of corrected fluorescence spectra.

\section{RESULTS AND DISCUSSION}

Figure 1 shows the comparison of the fluorescence spectra of PB and its para methylated derivative PB-M in two solvents of strongly different polarity. While in the nonpolar alkane solvent, there is only a narrow fluorescence band for $\mathrm{PB}$, a shoulder appears in acetonitrile which can be assigned to the emission from the CT state [1, 13-15]. In PB-M, however, this shoulder is absent in accordance with [15], evidencing that due to the slight reduction of the acceptor strength by the introduction of the methyl substituent the CT energy is lifted energetically such that it cannot be thermally populated any more.

Figure 2 shows the same comparison for the two compounds with increased donor acceptor strength, DM-PB and DM-PB-M. In this case, the short wavelength locally excited (LE) band is absent for all conditions, and the fluorescence spectra of the two compounds differ only marginally in the two solvents investigated. We can conclude that the CT is populated already in hexane for these compounds, and that the introduction of the methyl group on the benzene ring does not hinder its formation. 

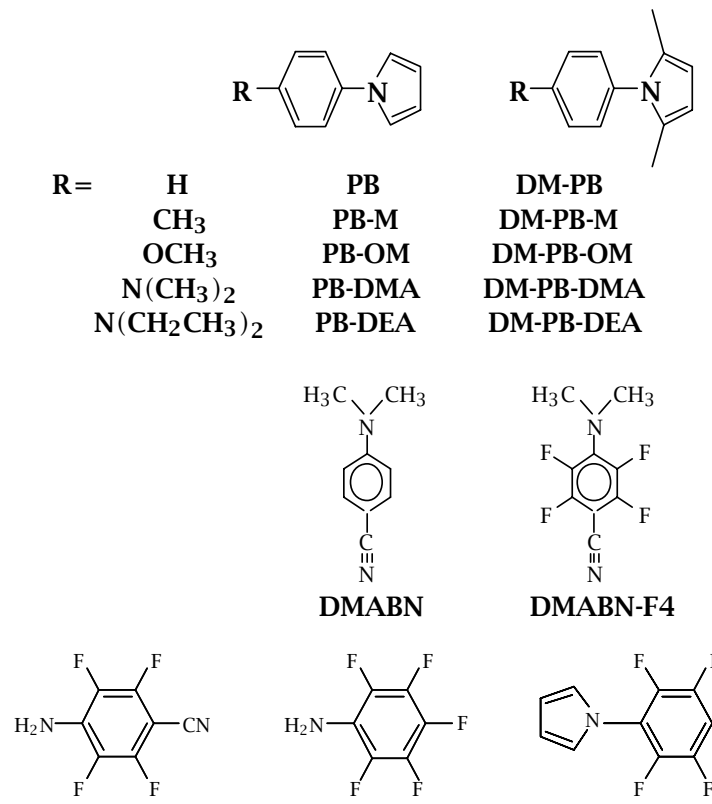

ABN-F4

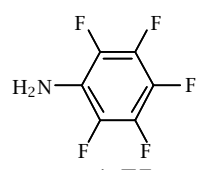

A-F5

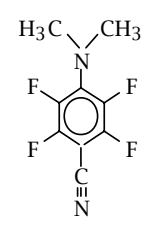

DMABN-F4

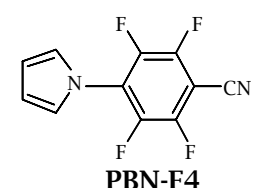

PBN-F4

Scheme 1. Structure and abbreviatin of the compounds investigated.
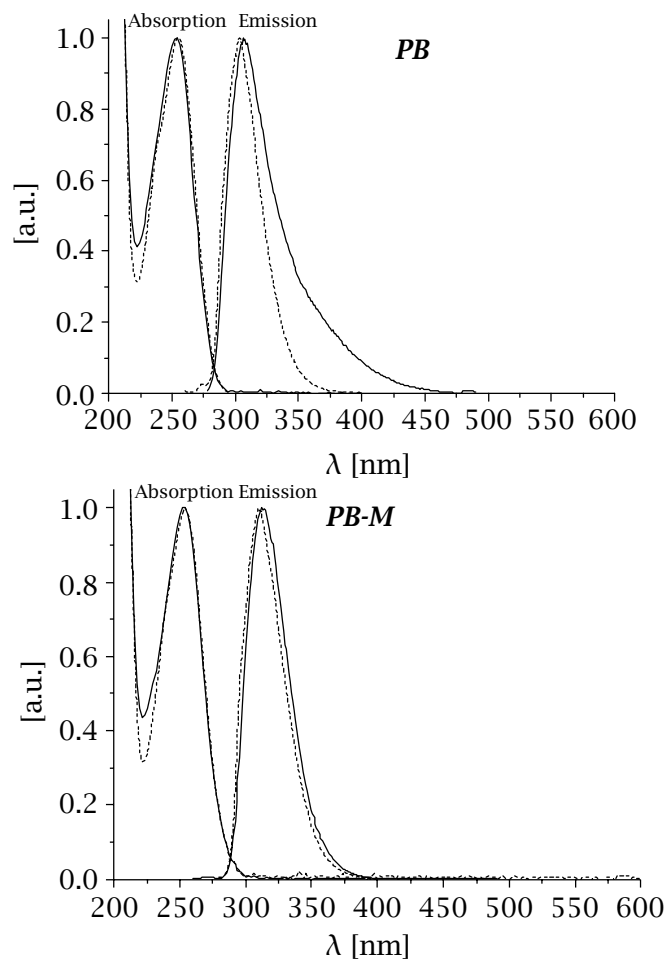

Figure 1. Fluorescence spectra of $\mathrm{PB}$ and $\mathrm{PB}-\mathrm{M}$ in hexane and acetonitrile at room temperature.

Figure 3 shows the spectra for the compounds where the donor strength of the para-substituent on
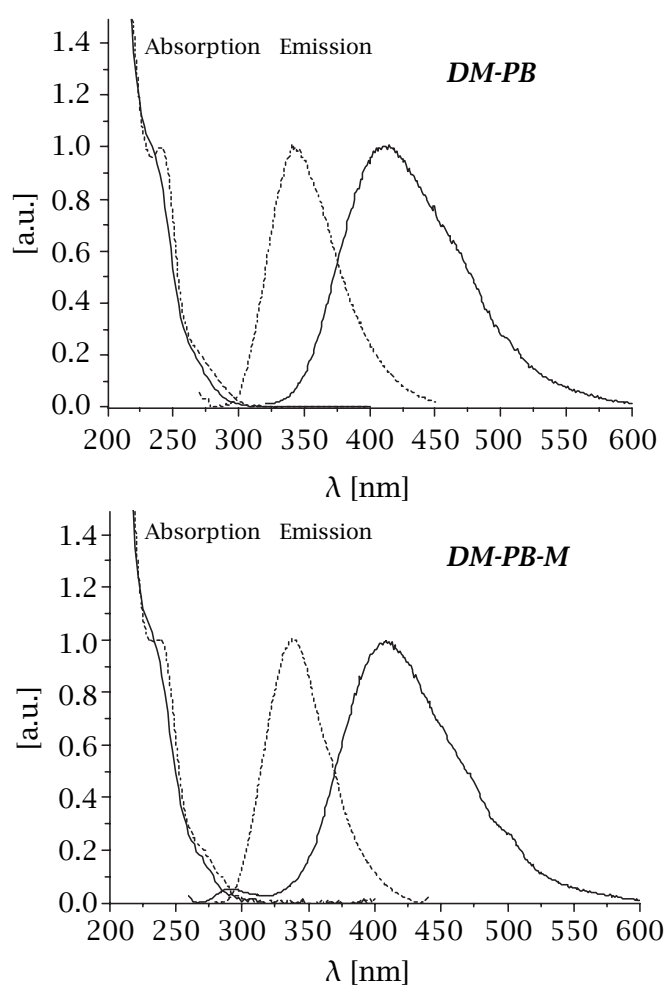

Figure 2. Fluorescence spectra of DM-PB and DM-PB-M in hexane and acetonitrile at room temperature.
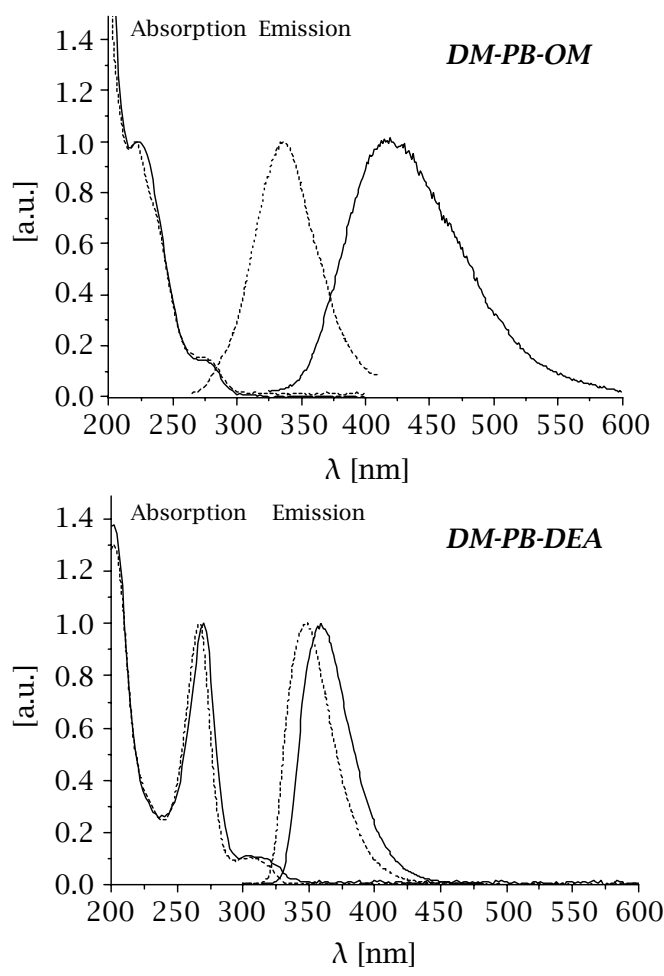

Figure 3. Fluorescence spectra of DM-PB-OM and DM-PBDEA in hexane and acetonitrile at room temperature. 

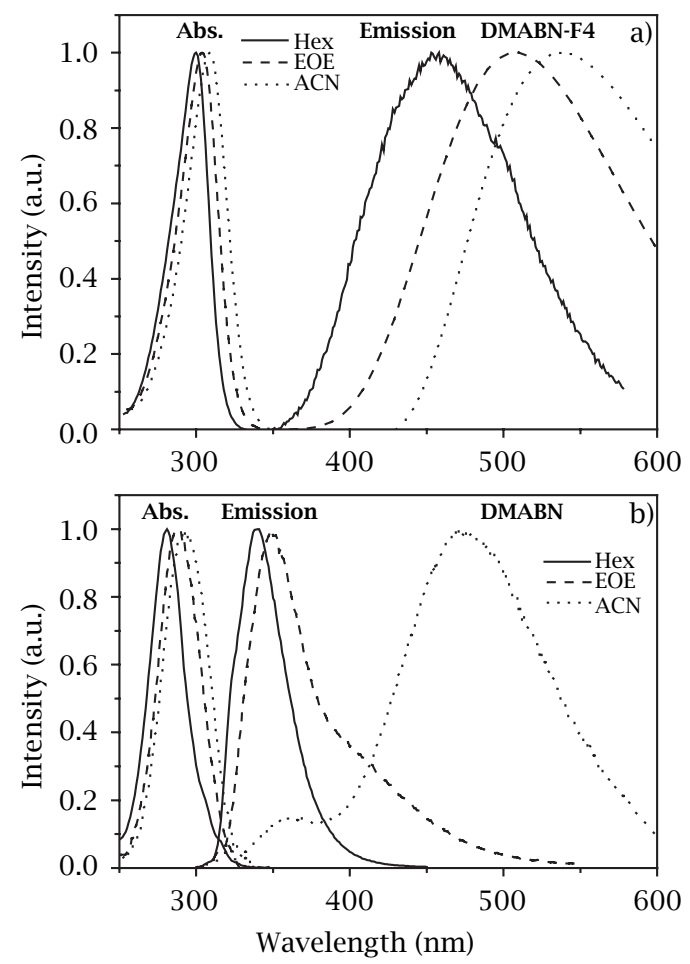

Figure 4. Absorption and fluorescence spectra of DMABNF4 and DMABN in hexane, diethyl ether and acetonitrile at room temperature.

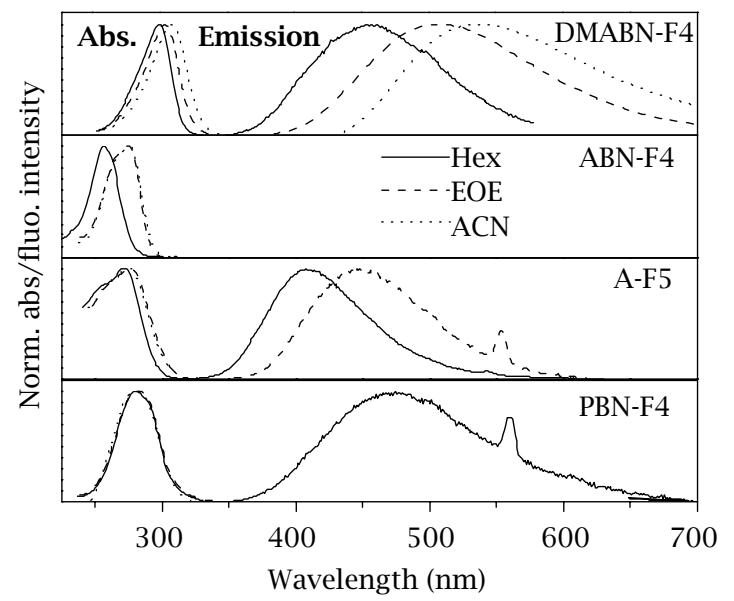

Figure 5. Absorption and fluorescence spectra of DMABNF4, ABN-F4, A-F5 and PBN-F4 in hexane, diethyl ether and acetonitrile at room temperature, excited in the main $a b$ sorption band. In the case of $\mathrm{ABN}-\mathrm{F} 4$ in all three solvents, for A-F5 in $\mathrm{ACN}$ and for PBN-F4 in diethyl ether and $\mathrm{ACN}$, the fluorescence intensity is too small for the measurement of a spectrum.

the phenyl ring is gradually increased. We can see that even for the methoxy group as a donor, there is pure
CT fluorescence in both solvents. This is remarkable in the sense that a CT state is normally not expected for a donor-donor-substituted benzene derivative. Only if we substitute with a still stronger donor, such as a dialkylamino group, the CT band is suppressed, and the normal LE band reappears.

Figure 4 shows the case where the acceptor strength in DMABN is increased by introducing four fluoro substituents. The spectra show clearly that already in alkane solvents, the CT state is populated, whereas in DMABN, similarly to $\mathrm{PB}$, more strongly polar solvents are necessary.

Figure 5 finally shows the results for further fluorinated derivatives of DMABN and PB. In all the cases, pure CT fluorescence is observed, evidencing that the LE states are situated energetically above the CT state for all of these compounds. Obviously, the four fluorine substituents are a powerful way of increasing the acceptor strength and switching the fluorescence from LE to CT behaviour. It is remarkable that even the amino group with its reduced donor strength leads to the redshifted and highly solvatochromic fluorescence spectra.

\section{CONCLUSIONS}

It is shown that the CT behaviour of aniline and phenyl pyrrole derivatives can be influenced by substituents in a predictable manner. Introduction of fluorine atoms into the acceptor part strongly enhances the tendency for populating the CT state. On the other hand, by a suitable balance of an increase of donor strength and a controlled decrease of acceptor strength it is possible to generate donor-donor-substituted benzene derivatives that populate a CT state.

\section{REFERENCES}

[1] Z. R. Grabowski, K. Rotkiewicz, and W. Rettig, Chemical Reviews 103 (2003), 3899.

[2] W. Weigel, W. Rettig, M. Dekhtyar, C. Modrakowski, M. Beinhoff, and A. D. Schlüter, J. Phys. Chem. A 107 (2003), 5941.

[3] Z. R. Grabowski, K. Rotkiewicz, A. Siemiarczuk, D. J. Cowley, and W. Baumann, Nouv. J. Chim. 3 (1979), 443.

[4] W. Rettig, Angew. Chem. 98 (1986), 969; Angew. Chem. Int. Edit. Engl. 25 (1986), 971.

[5] A. B. J. Parusel, Phys. Chem. Chem. Phys. 2 (2000), 5545.

[6] S. Zilberg and Y. Haas, J. Phys. Chem. A 106 (2002), 1.

[7] K. A. Zachariasse, Chem. Phys. Lett. 320 (2000), 8.

[8] K. A. Zachariasse, M. Grobys, T. von der Haar, A. Hebecker, Y. V. Il'ichev, Y. B. Jiang, O. Morawski, and W. Kühnle, J. Photochem. Photobiol., A: Chemistry 102 (1996), 59. 
[9] S. Murali, P. Changenet-Barret, C. Ley, P. Plaza, W. Rettig, M. M. Martin, and A. I. Tolmachev, Femtochemistry and Femtobiology: Ultrafast Events in Molecular Science (M. M. Martin and J. T. Hynes, eds.), Elsevier B.V., 2004, p. 323.

[10] S. Murali, V. Kharlanov, W. Rettig, A. I. Tolmachev, and A. V. Kropachev, submitted to J. Phys. Chem. A.

[11] S. Murali, W. Rettig, and A. I. Tolmachev, in preparation.
[12] A. Neubauer and W. Rettig, in preparation.

[13] W. Rettig and F. Marschner, Nouveau Journal de Chimie 7 (1983), 425.

[14] C. Cornelissen-Gude and W. Rettig, J. Phys. Chem. A 102 (1998), 7754.

[15] T. Yoshihara, V. A. Galiewsky, S. I. Druzhinin, S. Saha, and K. A. Zachariasse, Photochem. Photobiol. Sci. 2 (2003), 342. 


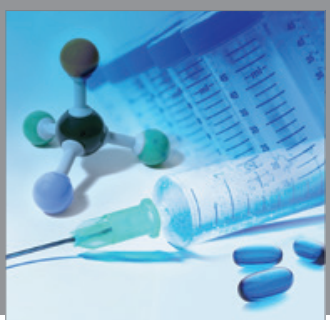

International Journal of

Medicinal Chemistry

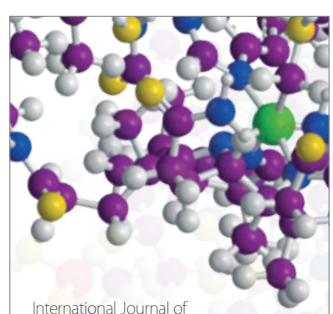

Carbohydrate Chemistry

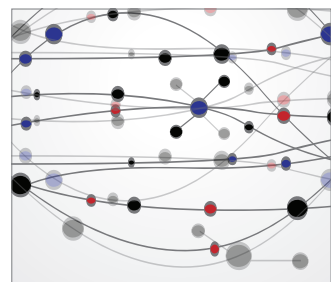

The Scientific World Journal
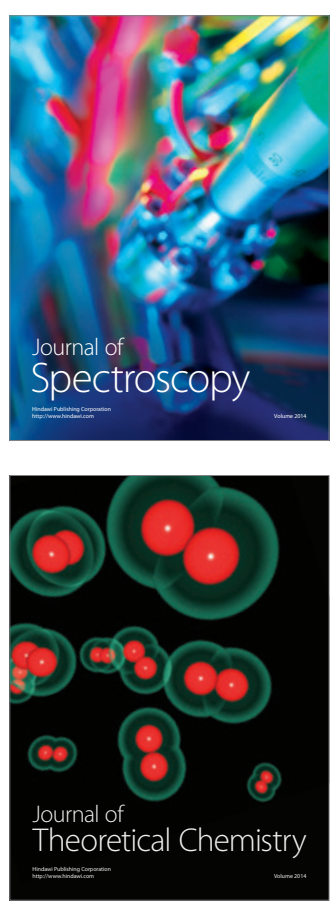
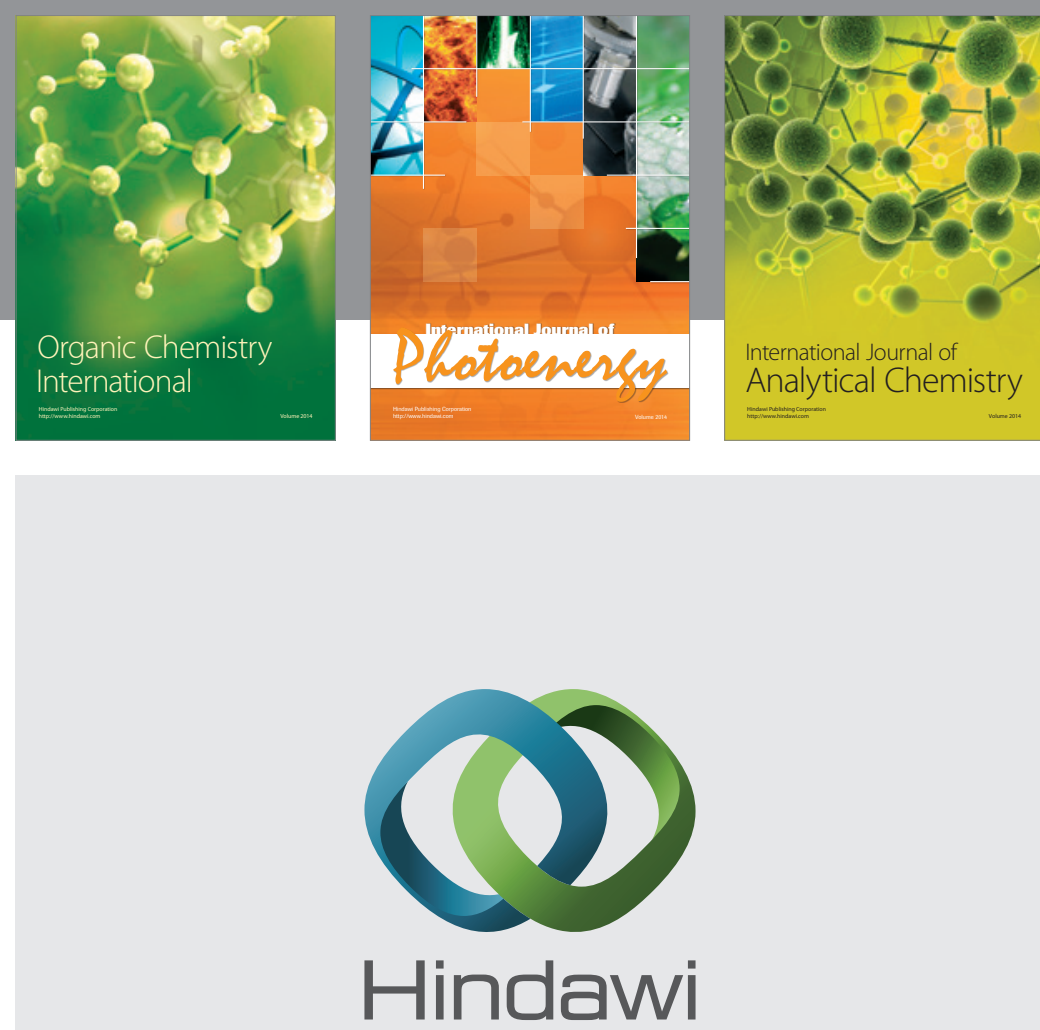

Submit your manuscripts at

http://www.hindawi.com
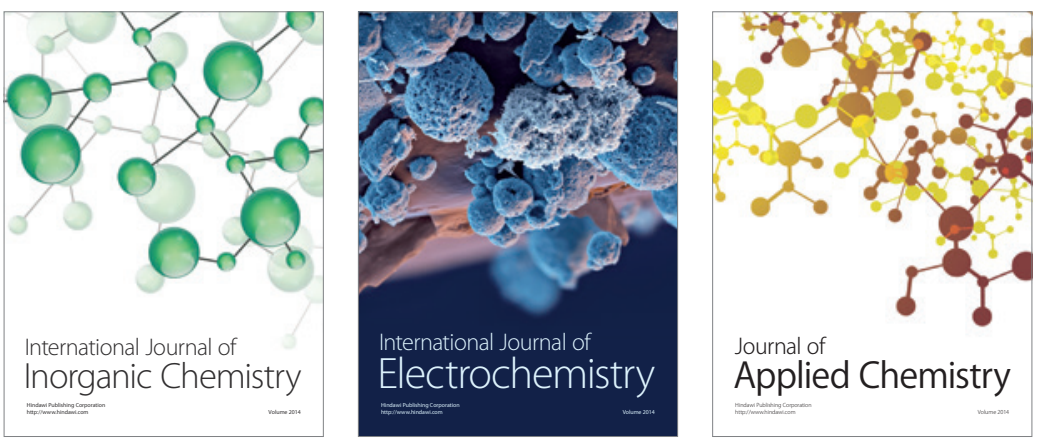

Journal of

Applied Chemistry
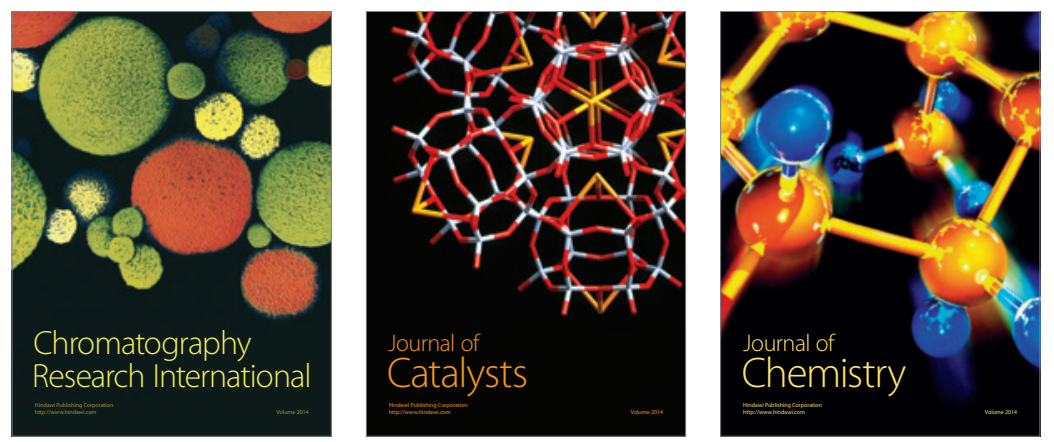
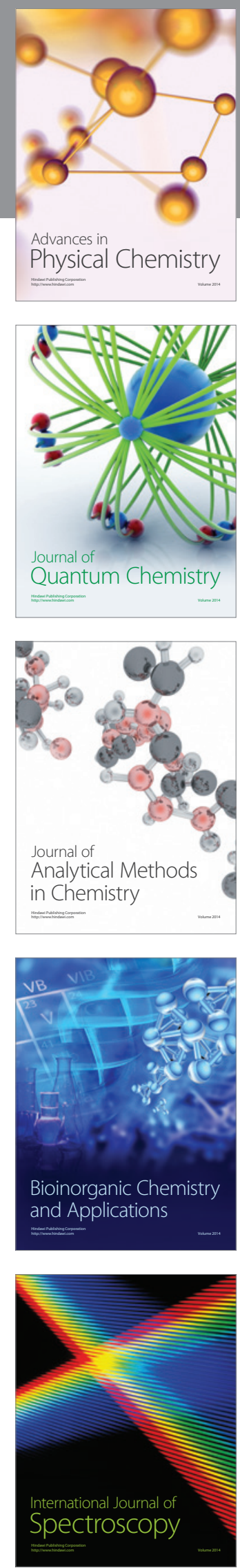